\title{
SÍNTESE E CARACTERIZAÇÃO DE FILMES NANOCOMPÓSITOS ENTRE ÓXIDO DE GRAFENO E AZUL DA PRÚSSIA VISANDO À APLICAÇÃO EM SENSORES E DISPOSITIVOS ÓPTICOS
}

\author{
L. C. MENDES 1 , C. B. de AQUINO², J. C. PIERETTI ${ }^{2}$, S. H. DOMINGUES 2 e E. NOSSOL ${ }^{3}$ \\ ${ }^{1}$ Universidade Federal de Uberlândia, Faculdade de Engenharia Química \\ 2 Universidade Presbiteriana Mackenzie, Mackgraphe - Graphene and Nano-Material \\ Research Center \\ ${ }^{3}$ Universidade Federal de Uberlândia, Instituto de Química \\ E-mail para contato: enossol@ufu.br
}

RESUMO - O grafeno, uma das formas alotrópicas do carbono, tem sido considerado o futuro da tecnologia em todo o mundo. A preparação de materiais derivados de grafeno tem merecido destaque nos últimos anos, tendo em vista as excelentes propriedades mecânicas, térmicas e elétricas desses materiais. Este projeto utiliza um filme de óxido de grafeno na preparação de filmes nanocompósitos contendo hexacionaferrato de ferro (III), conhecido como azul da Prússia. Através do presente projeto pretende-se desenvolver uma rota inovadora de preparação desse tipo de nanocompósito, através do método interfacial, com modulação das propriedades através do controle de morfologia, tamanho e dispersão do azul da Prússia sobre as folhas de óxido de grafeno. Os filmes obtidos foram caracterizados através das técnicas de espectroscopia por energia dispersiva de raios X (EDS ou EDX), técnicas de microscopia eletrônica de varredura e espectroscopia Raman. Essas técnicas mostraram a eficiência do método interfacial para obtenção dos filmes e revelaram características importantes para os diferentes materiais preparados, objetivando sua aplicação como sensor para diferentes analitos, além de componente ativo em dispositivos ópticos.

\section{INTRODUÇÃO}

O carbono é considerado um dos mais versáteis elementos químicos por apresentar diferentes alotropias, microtexturas, dimensionalidades e formas. Dentre os alótropos do carbono o grafite e o diamante ocupam posições de destaque. Com as possibilidades de variações no comportamento químico do carbono, juntamente com as propriedades inerentes de materiais nanométricos, há o favorecimento de formação de inúmeras estruturas que vem se apresentando como importante ferramenta de evolução na área tecnológica, entre as quais podemos citar os nanotubos de carbono, grafite, óxido de grafeno, óxido de grafeno reduzido, os fulerenos e o grafeno, cujas estruturas estão representadas na Figura 1.

O grafeno é um material bidimensional constituído por uma única camada de átomos de carbono com hibridização $\mathrm{sp}^{2}$, ligados entre si em hexágonos, onde cada vértice é ocupado por átomos de carbono, formando uma estrutura similar a de uma colméia. Esse tipo de configuração proporciona a esse material propriedades únicas e de grande interesse na comunidade cientifica mundial. Trata-se de um material extremamente resistente, flexível, leve, com elevada área superficial $\left(2630 \mathrm{~m}^{2} / \mathrm{g}\right)$, alta condutividade térmica $\left(\mathrm{K}=5 \times 10^{3}\right.$ 


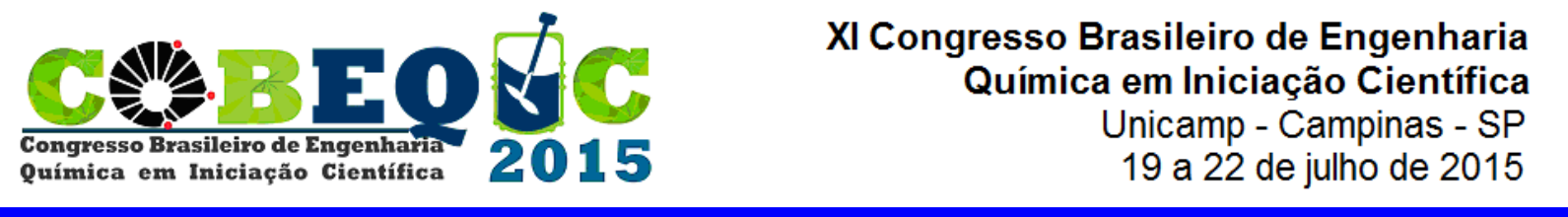

$\left.\mathrm{Wm}^{-1} \mathrm{~K}^{-1}\right)$ e elétrica $\left(\sigma=64 \mathrm{mS} \mathrm{cm}^{-1}\right)$, é quase totalmente transparente, além de excelentes propriedades mecânicas, segundo Martín e Escarpa (2014).

Figura 1 - Estruturas do grafeno (a), fulereno (b), nanotubo de carbono (c), grafite (d), óxido de grafeno (e) e óxido de grafeno reduzido (f).

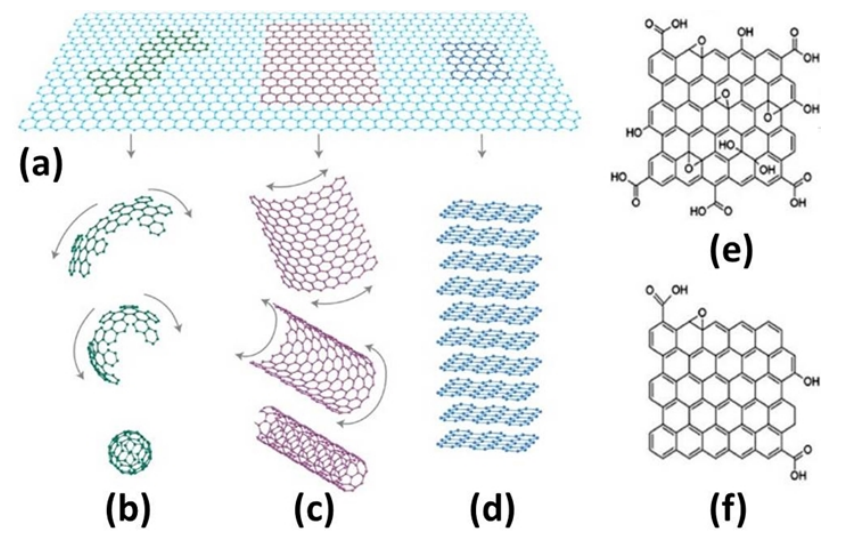

Entre os materiais derivados do grafeno que vem recebendo destaque cientifico está o óxido de grafeno (do inglês graphene oxide - GO). Além de ser um precursor para a produção de grafeno em larga escala, ele também possui, ao contrário do grafeno, uma ótima dispersibilidade em água, e a presença de grupos funcionais na superfície. O modelo mais aceito para a estrutura do GO apresenta duas regiões distintas na folha: o plano basal dominado pela presença de grupos hidroxila e epóxido; e, os planos de borda, onde encontram-se principalmente grupos carboxílicos ou carboxilatos. Nessa rede hexagonal de carbonos, a região não-funcionalizada apresenta hibridização $\mathrm{sp}^{2}$, e a funcionalizada $\mathrm{sp}^{3}$. Entre as aplicações para o GO destacam-se a sua utilização e supercapacitores flexíveis (El-Kady et al., 2013), armazenamento de dados (Li et al., 2013) e catálise (Pyun et al., 2011).

Um dos compostos mais estudados na preparação de nanocompósitos é o azul da Prússia (AP), de fórmula $\mathrm{Fe}_{4}\left[\mathrm{Fe}(\mathrm{CN})_{6}\right] \cdot \mathrm{xH}_{2} \mathrm{O}$, cuja estrutura cristalina é formada por íons férricos $[\mathrm{FeIII}]$ e ferrosos [FeII] alternando-se nos sítios de um retículo cúbico, como mostrado na Figura 2-A. Tendo em vista as limitações quanto às aplicações do AP devido à estabilidade destes hexacianometalatos, uma das formas mais promissoras de se aumentar essa estabilidade, além de propiciar efeitos sinérgicos no material final, segundo Li et al. (2007), consiste na preparação de nanocompósitos entre o AP e materiais carbonáceos (Figura 2-B).

A obtenção de nanocompósitos entre AP e materiais derivados do grafeno, como o GO, vem propiciando a observação de propriedades diferenciadas nesses materiais, como efeitos eletrocatalíticos acentuados (Martín et al., 2014) e aumento da estabilidade do nanocompósito (Cao et al., 2010). Entre as principais aplicações para esse material estão o uso em baterias de íon-lítio (Kumar et al., 2014), células a combustível (Wang et al., 2013), dispositivos eletrocrômicos (Yang et al., 2014) e sensores para diferentes substâncias (Cao et al., 2010, onde para os compósitos GO/AP destaca-se o uso na determinação de $\mathrm{H}_{2} \mathrm{O}_{2}$, como pode ser visto na Figura 2-C. 


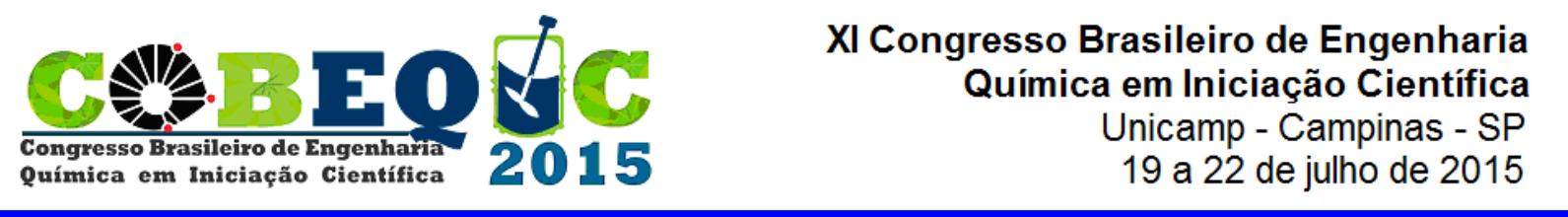

Figura 2 -Estrutura ideal cúbica de face centrada do AP (A). Imagem de microscopia eletrônica de varredura de um nanocompósito GO/AP (B) e sua atividade para determinação de $\mathrm{H}_{2} \mathrm{O}_{2}$, mostrando voltamogramas cíclicos antes (C-a) e após (C-b) a adição de $\mathrm{H}_{2} \mathrm{O}_{2}$.
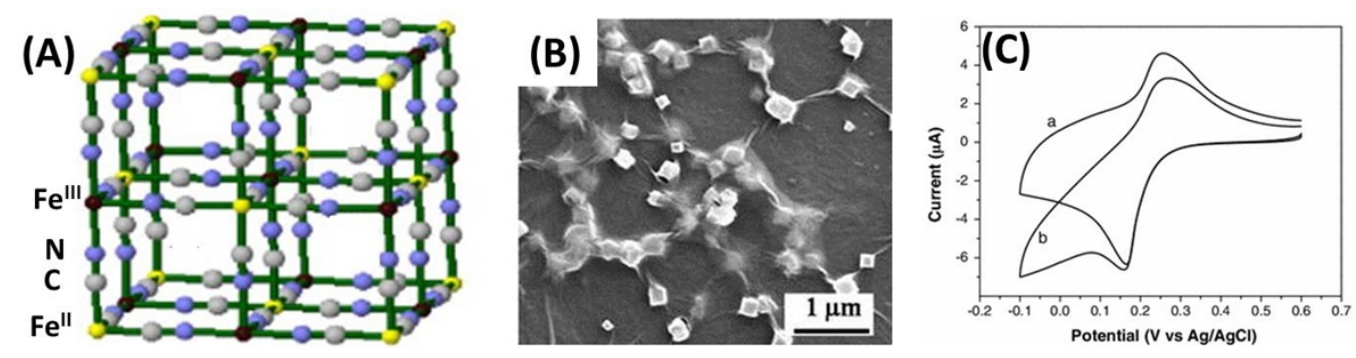

\section{OBJETIVOS}

O presente trabalho tem como objetivos principais a síntese de filmes finos nanocompósitos do tipo óxido de grafeno/azul da Prússia, com estudo de suas propriedades, visando à aplicação em sensores e dispositivos ópticos.

\section{MATERIAIS E MÉTODOS}

\subsection{Preparação do Óxido de Grafeno}

A reação de obtenção de óxido de grafeno foi realizada duas etapas. Na primeira o grafite foi oxidado pelo agente oxidante $\mathrm{Mn}_{2} \mathrm{O}_{7}$, passado o período de reação entre o grafite e o agente oxidante, a reação é então diluída com adição de $\mathrm{H}_{2} \mathrm{O}$, seguido da adição de $\mathrm{H}_{2} \mathrm{O}_{2}$. Posteriormente o sólido formado é filtrado, lavado e seco em estufa. O material seco é agora denominado óxido de grafite (Gr-O). Após obtenção do Gr-O este é disperso em água através de banho de ultrassom formando então dispersões de óxido de grafeno (GO).

\subsection{Preparação dos Filmes GO/AP Pelo Método Interfacial}

A síntese dos filmes GO/AP foi realizada, em duas etapas, pelo método interfacial. Primeiramente $0,01 \mathrm{~g}$ de $\mathrm{GO}$ foram adicionadas a $20 \mathrm{~mL}$ de água, com posterior agitação magnética por $24 \mathrm{~h}$ e dispersão em banho de ultrassom por 30 minutos. Ao término desse período, a dispersão de GO foi colocada em um balão volumétrico com capacidade para 50 $\mathrm{mL}$ contendo a fase orgânica composta por $20 \mathrm{~mL}$ de clorofórmio. Esse sistema foi mantido sobre agitação por $24 \mathrm{~h}$. Posteriormente, a agitação magnética foi interrompida e a fase aquosa lavada sucessivamente com $\mathrm{H}_{2} \mathrm{O}$ destilada, e substituída por $20 \mathrm{~mL}$ de uma solução ácida ( $\mathrm{pH} 2)$ contendo $\mathrm{K}_{3}\left[\mathrm{Fe}(\mathrm{CN})_{6}\right]\left(0,5 \mathrm{mmol} \mathrm{L}^{-1}\right)$ e $\mathrm{FeCl}_{3}\left(0,5 \mathrm{mmol} \mathrm{L}^{-1}\right)$.

Após a formação de um filme interfacial, o clorofórmio foi substituído por $20 \mathrm{~mL}$ de ciclohexano, e o sistema submetido à agitação magnética por mais $1 \mathrm{~h}$. Decorrido esse tempo foram adicionados $10 \mu \mathrm{L}$ de $\mathrm{H}_{2} \mathrm{O}_{2}\left(0,5 \mathrm{~mol} \mathrm{~L}^{-1}\right)$, com o sistema permanecendo sob agitação durante 3 horas, com subsequente lavagem da fase aquosa. O material formado no balão foi adicionado a um béquer contendo água destilada. A remoção do filme ocorreu através da colocação do substrato no recipiente onde foi efetuada a preparação do filme (transferência, lavagem). $\mathrm{O}$ filme foi removido com o auxílio de uma haste metálica previamente fixada no substrato de interesse, onde houve a possibilidade de erguê-lo, recolhendo assim o filme. 


\section{RESULTADOS E DISCUSSÃO}

\subsection{Espectroscopia Raman}

A Figura 3 apresenta os espectros Raman referentes ao filme de $\mathrm{GO}$ e ao nanocompósito GO/AP. No espectro obtido a partir do filme de GO observa-se a presença das bandas D e G, em 1345 e $1600 \mathrm{~cm}^{-1}$, respectivamente. A banda D evidencia principalmente as imperfeições estruturais do GO, provenientes de efeitos de bordas, impurezas, heteroátomos e carbonos com hibridização $\mathrm{sp}$ e $\mathrm{sp}^{3}$. A banda $\mathrm{G}$ caracteriza os átomos de carbono com hibridização $\mathrm{sp}^{2}$ (Dresselhaus et al., 2010).

Figura 3 - Espectros Raman dos filmes nanocompósitos GO e GO/AP.

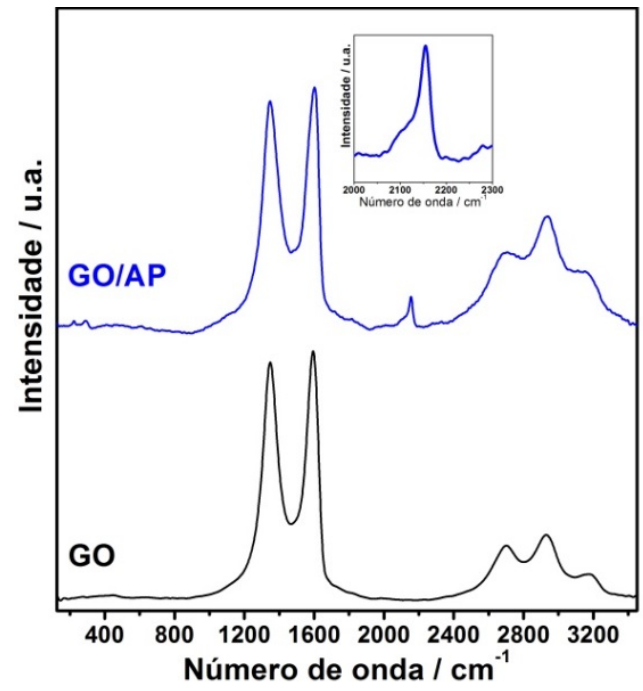

O espectro do nanocompósito GO/AP mostra, além das bandas D e G, a presença de bandas características do AP, encontradas na região entre 500 a $600 \mathrm{~cm}^{-1}$, atribuídas aos modos vibracionais Fe-N, Fe-C, Fe-CN ou Fe-NC. Adicionalmente, nota-se na Figura 4 (em detalhe) duas bandas em 2105 e $2155 \mathrm{~cm}^{-1}$, atribuídas ao estiramento $\mathrm{C} \equiv \mathrm{N}$ ligado ao $\mathrm{Fe}$ (II) e Fe(III), respectivamente, confirmando a formação do AP (Mažeikienè et al., 2011).

\subsection{Microscopia eletrônica de varredura e EDX}

Os filmes de GO e GO/AP também foram analisados através da técnica de microscopia eletrônica, com os resultados apresentados na Figura 4. As imagens referentes ao filme de GO mostram um material de certa rugosidade e que cobre uniformemente grande proporção do substrato (Figuras 4-a e 4-b). Também pode-se afirmar que o fime obtido possui uma espessura bastante reduzida. As Figuras 4-c e 4-d mostram as imagens obtidas para o filme nanocompósito GO/AP, onde nota-se a presença de um grande número de nanopartículas de AP distribuídas ao longo das folhas de GO, atestando a eficácia do método interfacial para preparação desse material. Outra informação importante observada através das imagens de MEV para o nanocompósito é que as nanopartículas se formam preferencialmente nas folhas de GO, indicando uma forte interação entre esses materiais. Essa característica é importante em futuras aplicações envolvendo o nanocompósito preparado. 


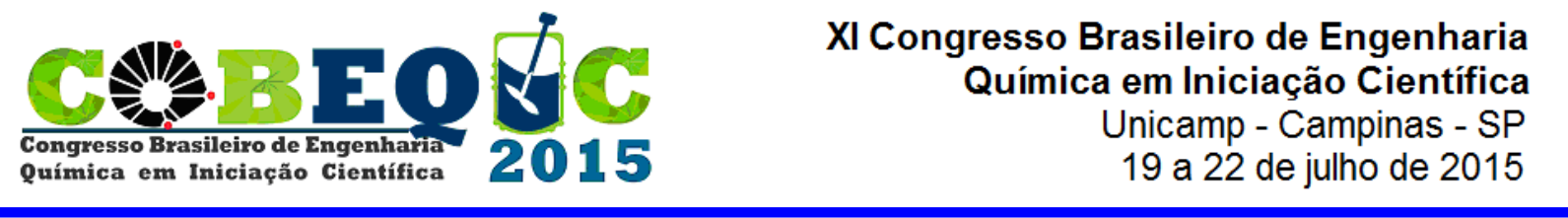

O mapeamento elementar do filme de GO e do nanocompósito GO/AP foi realizado utilizando análise dispersiva de raios X (EDX) e está apresentado na Figura 4.

Figura 4 - Imagens de MEV referentes ao filme de GO (a e b) e o nanocompósito $\mathrm{GO} / \mathrm{AP}$ (c e d). EDX do filme de GO (e) e do nanocompósito GO/AP
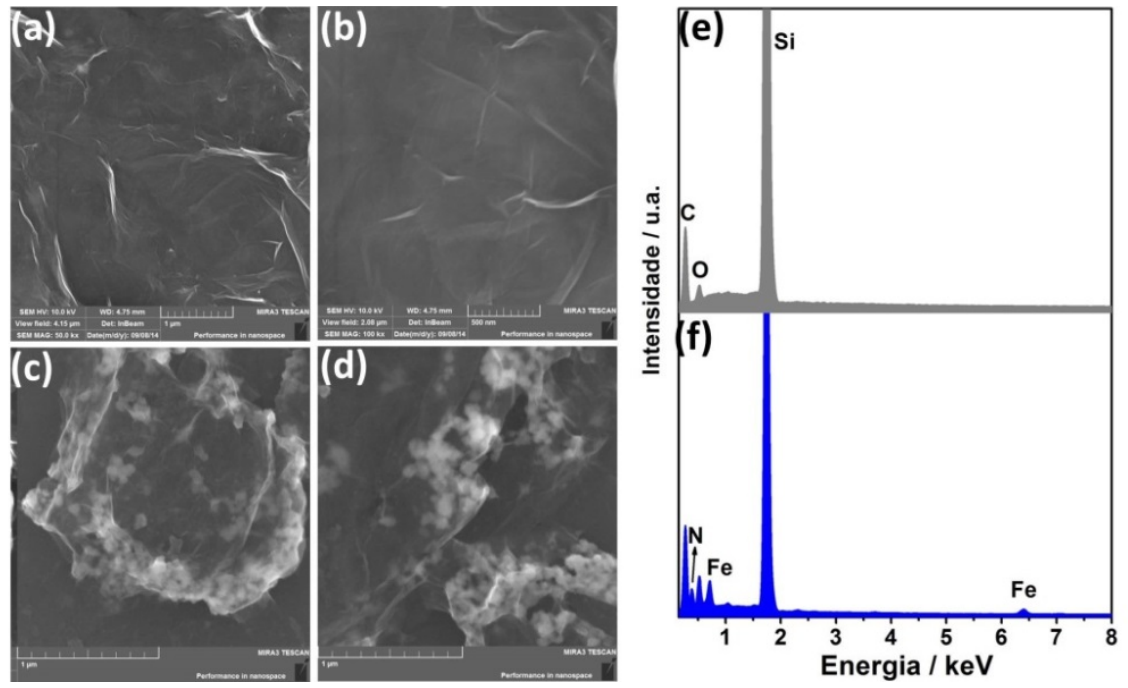

O espectro referente ao filme de GO (Figura 4-e) mostrou picos referentes a presença de $\mathrm{C}(0,26 \mathrm{keV})$ e oxigênio $(0,51 \mathrm{keV})$, e que são elementos constituintes desse material carbonáceo. O nanocompósito GO/AP (Figura 4-f) presenta, além dos picos mencionados anteriormente, sinais atribuídos a $\mathrm{N}(0,38 \mathrm{keV})$ e $\mathrm{Fe}(0,71$ e $6,40 \mathrm{keV})$, confirmando a formação do AP no material.

\section{CONCLUSÕES}

O método interfacial se mostrou como uma rota eficaz para preparação de nanocompósitos entre GO e AP, com a obtenção de filmes finos, homogêneos e sem impurezas. As técnicas usadas confirmaram a formação do material, indicando uma forte interação entre os componentes. Esse nanomaterial se apresenta como um candidato promissor em aplicações envolvendo a construção de sensores e dispositivos ópticos.

\section{REFERÊNCIAS}

CAO, L., LIU, Y., ZHANG, B., LU, L., In situ Controllable Growth of Prussian Blue Nanocubes on Reduced Graphene Oxide: Facile Synthesis and Their Application as Enhanced Nanoelectrocatalyst for $\mathrm{H}_{2} \mathrm{O}_{2}$ Reduction. ACS Appl. Mater. Interfaces, v. 2, p.2339-2346, 2010.

DRESSELHAUS MS, JORIO A., HOFMANN M., DRESSELHAUS G., SAITO R., Perspectives on carbon nanotubes and graphene Raman spectroscopy. Nano Lett., p.751, 2010 .

EL-KADY, M. F., KANER, R. B., Scalable fabrication of high-power graphene microsupercapacitors for flexible and on-chip energy. Nat. Commun., v. 4, p.1475, 2013. 
H. N. LIM, N. M. HUANG, C. H. CHIA, I. HARRISON, Advanced Topics in Crystal Growth, Croatia: In Tech Publication, 2013.

JOHN M. CHALMERS, HOWELL G. M. EDWARDS, MICHEL D. HARGREAVES, Infrared an Raman Spectroscopy in Forensic Science. Wiley, 2012.

KO, J. H., YEO, S., PARK, J. H., CHOI, J., NOH, C., SON, S. U., Graphene-based electrochromic systems: the case of Prussian Blue nanoparticles on transparent graphene film. Chem. Commun., v. 48, p.3884-3886, 2012.

KUMAR, A., PETERS, E. C., BURGHARD, M., Self-assembled magnetic nanoparticles of Prussian blue on graphene. RSC Adv., v. 4, p.18061-18064, 2014.

LI, Z., CHEN, J., LI, W., CHEN, K., NIE, L., YAO, S., Improved electrochemical properties of prussian blue by multi-walled carbon nanotubes. J. Electroanal. Chem ., v. 603, p.5966, 2007.

MARTÍN, A., ESCARPA, A., Graphene: The cutting-edge interaction between chemistry and electrochemistry. TrAC, Trends Anal. Chem., v. 56, p.13-26, 2014.

MAŽEIKIENĖ, R., NIAURA, G., MALINAUSKAS, A., Electrocatalytic reduction of hydrogen peroxide at Prussian blue modified electrode: An in situ Raman spectroelectrochemical study. J. Electroanal. Chem ., v. 660, p.140-146, 2011.

NOSSOL, E., GORGATTI ZARBIN, A. J., Transparent films from carbon nanotubes/Prussian blue nanocomposites: preparation, characterization, and application as electrochemical sensors. J. Mater. Chem., v. 22, p.1824-1833, 2012.

PYUN, J., Graphene Oxide as Catalyst: Application of Carbon Materials beyond Nanotechnology. Angew. Chem., Int. Ed., v. 50, p.46-48, 2011.

WANG, X.-J., KRUMEICH, F., NESPER, R., Nanocomposite of manganese ferrocyanide and graphene: A promising cathode material for rechargeable lithium ion batteries. Electrochem. Commun., v. 34, p.246-249, 2013.

YANG, H., LI, H., ZHAI, J., SUN, L., ZHAO, Y., YU, H., Magnetic prussian blue/graphene oxide nanocomposites caged in calcium alginate microbeads for elimination of cesium ions from water and soil. Chem. Eng. J. (Amsterdam, Neth.), v. 246, p.10-19, 2014. 